\title{
Aprender entre dos culturas: Estudio de un caso de escolarización bilingüe para hijos de emigrantes
}

\author{
Antonio Guerrero
}

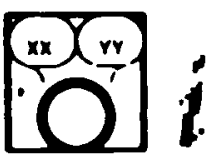

El tratamiento escolar que recibe la emigración de nuestro pais en Europa por parte de la Administración española es poco conocido. En este artículo, de forma resumida, el autor repasa alguno de los problemas de la "segunda generación» de emigrantes desde el punto de vista de su escolarización. El trabajo realizado en el colegio "Portobello" de Londres según las técnicas de la observación participante presenta las dificultades para desarrollar una educación bilingüe de calidad.

Las líneas que siguen a continuación exponen, de una manera abrevia$\mathrm{da}$, las principales conclusiones de una investigación desarrollada en el colegio bilingüe para hijos de emigrantes españoles «Vicente Cañada-Blanch». Conocido entre la colonia española como «Portobello», por la calle londinense donde está ubicado, el colegio es uno de los dos centros bilingües que la Junta de Promoción Educativa tiene en la actualidad (el otro está en París) y presenta la ventaja de reunir en su interior las diferentes formas de escolarización que ofrece el sistema educativo en la emigración (SEE): tanto enseñanzas integradas como complementarias.

\section{CONSIDERACIONES METODOLOGICAS}

Allí, mediante la observación participante, fueron estudiados una serie de grupos y unidades didácticas, tanto en inglés como en castellano. El muestreo de las clases y grupos a observar tuvo en cuenta, también, los diferentes niveles educativos (Primaria, Secundaria y Bachillerato). Al mismo tiempo, se incluyeron tanto las clases de las materias que -a priori al menos- más directamente estaban relacionadas con la cultura y la lengua, tales como «English», «Social Studies», Historia o Lengua, como aquellas 
otras que, como Matemáticas o Educación Física, parecían en principio más neutras. Con todo, la mayoría de las clases observadas fueron de los grupos de edad entre 12 y 16 años, por ser la fase terminal del Colegio y, en principio, de resolución del dilema entre los dos sistemas educativos. En tales clases, la observación se centró en las interacciones entre los propios alumnos y alumnas, y las de éstos con el profesor o profesora, tanto en los aspectos lingüísticos, como los de transmisión del «arbitrario cultural». Paralelamente, y en cuanto a métodos discretos se refiere, se recogió información procedente de fuentes tan dispares como las listas de cursos (para ver los matrimonios «mixtos", por ejemplo), las rutas de autobuses (para localizar los núcleos de residencia más importantes, así como el radio de acción del centro); notas, avisos y anuncios de los tablones; murales, recortes de prensa y trabajos colgados en las paredes del centro, así como las pintadas de los pupitres - quizá los diálogos más apasionados-y, en fin, los ficheros del centro.

Las entrevistas fueron de dos tipos: unas informales, aprovechando un encuentro o una situación, y otras formales, establecidas de antemano y con guión previo. De éstas, algunas fueron grabadas en magnetofón. Las entrevistas formales sólo tuvieron lugar con profesores/as y al final del trabajo de campo. En gran medida, adquirieron el papel de "triangular» (Denzin, 1970) algunas de las explicaciones halladas en el análisis. Otra forma de «triangulación», la llamada «respondent validation" (Ball, 1984), se llevó también a cabo una vez concluido un primer borrador de la memoria. Este fue enviado al claustro y discutido con las profesoras y profesores del nivel de secundaria. Tal "validación» no fue, sin embargo, posible con el alumnado por razones de fecha (por entonces, ya había acabado el curso), pero un ensayo parcial tuvo lugar con alumnos y alumnas en una de las clases de $3 .^{\circ}$ de "Complementarias» ${ }^{1}$.

A continuación, pues, sigue una interpretación de los datos recogidos en "Portobello", a modo de reseña "cariñosa" de los avatares y conflictos —en minúsculas y voz baja- que supone aprender y crecer entre dos culturas.

\section{EL SEE: BICULTURALISMO, COMPLEMENTARIEDAD Y CONVERTIBILIDAD}

La inicial cobertura estatal a la emigración, caracterizada por ser un aparato de vigilancia a la vez que «cordón sanitario» contra contaminaciones democráticas, cambió al comienzo de los 70 . El cambio fue hacia una estructura asistencial para una comunidad, como la española en Europa, que había dejado de ser temporal para establecerse en los países de acogida. De la barraca de soltería al piso, se produjo la reunificación familiar y la cría de la descendencia. Una serie de colonias españolas fueron así formándose en los barrios industriales de muchas ciudades europeas. Su sentido de comunidad se vio reforzado por un continuo y contumaz rechazo de la población autóctona. Los emigrantes españoles, hombres y mujeres, niñas y niños, tuvieron que soportar la discriminación que se les propinaba en base a una diferenciada visibilidad física (color del pelo, estatura, etc.) y cultural (forma de vestir o caminar por las calles - hombres delante, mujeres de- 
trás) e, incluso, audibilidad (acento) y que les suponía verse encasillados y dirigidos hacía los peores trabajos, las peores viviendas y los más desatendidos servicios. Paralelamente, la naciente crisis económica les forzó a prolongar indefinidamente su estancia, al retrasarse "sine die» las posibilidades de retorno.

Como parte central de ese aparato asistencial, era necesaria, sin duda, una institución formal que mantuviese, más allá de contactos «folklóricos» por Navidad, los lazos culturales con España de forma permanente, al tiempo que aliviase la situación de acoso de tales comunidades. De crucial importancia en esta labor era el reforzamiento de la identidad cultural para mantener abierta la posibilidad del retorno y la necesidad de ahorrar y de enviar ese ahorro, en forma de «remesas» de divisas, a España. Si las Casas de España van a desempeñar esa tarea de agentes culturales con las personas adultas, el sistema educativo va a ser el principal agente para mantener la identidad cultural en sus hijas e hijos, guardándolos dentro del idioma, la cultura y los conocimientos escolares oficiales.

En palabras de uno de sus responsables (Soler, 1984: 24), dicho sistema pretendía responder a la necesidad de una educación integrada que permita evitar el aislamiento social y la creación de "guetos». De esta loable postura cabría deducir que lo más conveniente es un «sistema integrado» de enseñanzas complementarias de «lengua y cultura españolas». Esto es, enseñar Lengua, Geografía e Historia de España por profesores españoles, dentro del horario y de las aulas de las escuelas locales donde esos niños y niñas asistiesen. Sin embargo, problemas técnicos y legales han hecho que este planteamiento de integración bicultural sólo represente, en el conjunto del SEE, una pequeña proporción y en una minoría de países. La mayoría del alumnado asiste a las llamadas «Clases Complementarias de Lengua y Cultura Española», unas clases impartidas por profesores españoles, seleccionados por concursos de méritos entre los del Cuerpo correspondiente, en locales arrendados a las autoridades locales y fuera del horario escolar, unas veces en sábado y otras por las tardes. Se ve pues, fácilmente, que vienen a alargar una de por sí ya exhausta jornada para esos niños, con desplazamientos a veces distantes del domicilio. El trabajo extra, incluyendo los dobles deberes y el doble horario, contribuyen a aumentar su cansancio. Comoquiera que, en la gran mayoría de los casos, van a dichas clases obligados por sus padres, un conflicto entre padres e hijos, surge, con manifestaciones en una irregular asistencia e importantes abandonos. Con esa actitud, por otra parte, esos alumnos parecen estar mostrando sus preferencias por la enseñanza de sus -en la mayoría de los casos- países de nacimiento. En dicha enseñanza encuentran una "clasificación» más débil (Bernstein, 1975) entre sus actividades escolares y su entorno, una relación menos aislada entre conocimiento escolar y sociedad. Sobre todo en relación a los contenidos que les son transmitidos en las «clases complementarias", en locales mal acondicionados, y sobre un país al que apenas conocen por sus vacaciones estivales. Una manifestación excepcional del SEE la constituyen los dos colegios bilingües ya citados de París y Londres. A primera vista es difícil no caer en la tentación de clasificarlos en uno de los dos extremos del dilema arriba mencionado, concretamente como guetos. Por lo menos, y de acuerdo con el estudio realizado en «Portobello", el hecho de que sus estudiantes lo consideren su «hogar español", "lo mejor que 
tenemos los chicos españoles para defendernos de las agresiones exteriores", parece reforzar tal inclinación. En cualquier caso, estos colegios parecen estar adaptados para aquellos padres con mayor inclinación hacia el retorno, pero que, no obstante, quieren mantener abierta la posibilidad de quedarse: dos lenguas, dos titulaciones, dos países, dos opciones. Correlativamente, las enseñanzas «Complementarias» parecen más adecuadas para aquellos padres que están decididos a permanecer, al menos a medio plazo, pero que no quieren tener cerrada la posibilidad del retorno.

Sea como fuere, a la hora de establecer una relación entre la emigración en su conjunto y el SEE, un elemento sustantivo parece ser la clave en cualquiera de las dos posibilidades citadas: las titulaciones que se obtienen en el sistema local y en el SEE y su convalidación por su equivalente en España. El propio SEE, a través de su red territorial en el país de emigración, facilita el «Libro de Escolaridad» que da validez automática a las materias, cursos y niveles conseguidos tanto en el colegio local como en las clases de "complementarias", en el nivel correspondiente del sistema educativo español.

Tal «credencialismo», como diría Collins (1979), no tiene por qué ser visto como algo específico de la emigración, ya que no difiere del que otros grupos sociales muestran aquí o allá. Lo significativo en este caso estriba en que se trata de convertir el "capital académico» (Bourdieu y Passeron, 1977) que los hijos e hijas de emigrantes han obtenido en el país de residencia, en un capital nacional válido en España, el país de un posible retorno. Es algo similar a las remesas enviadas por sus padres a las cuentas de ahorro «en pesetas convertibles». Convalidación y convertibilidad se hacen la una homóloga de la otra. Se podría decir, entonces, que el SEE está desempeñando el mismo papel que las agencias de los bancos y cajas de ahorro enclavadas en los países de emigración: canalizar y cambiar el capital cultural extranjero en otro de «curso legal» en España. Una muestra de ello está en el gran interés que en la emigración se ha tenido siempre por las convalidaciones de estudio, tema de continua reivindicación ante la Administración española.

\section{El colegio «Cañada-Blanch»: Interacciones en su interior}

El alumnado de «Portobello» está constituido por hijos e hijas de trabajadores/as españoles en Inglaterra, nacidos mayoritariamente allí (El $83,4 \%$ de los 534 alumnos/as del curso 1986-87 había nacido en Londres. Del 16,4 nacido en España, el $10 \%$ lo había hecho en La Coruña). Un primer elemento que llama la atención es que su distribución por cursos no es, en modo alguno, regular. Hay menos matrícula en los cursos iniciales (Párvulos y $1 .^{\circ}$ de EGB) y en los finales $\left(10^{\circ}\right.$ y $2 .^{\circ}$ de BUP). Así, mientras la media de alumnos en cada curso de EGB es de 52,3 (divididos en 2 grupos), en Párvulos había sólo 34, en 1..$^{\circ}$ de BUP, 38 y en 2., 20 (Datos del curso 1986-87). La reducción en el número de entradas en los primeros cursos (últimas promociones) hace pensar en algunas posibles explicaciones: a) una lenta pero continua extinción de la llamada «segunda generación" sin dar aún paso a una «tercera»; b) un proceso de retorno a España, también reducido pero continuo, y c) una integración paulatina en el país de acogida, en la vecindad y en los lugares de trabajo, que haga innecesario utilizar ese «área protegida» que es «Cañada-Blanch». El incremento del nú- 
mero de hijas e hijos de matrimonios' mixtos en la últimas promociones, cuatro veces superior a las primeras, parece dar fuerza a esta tercera posibilidad.

En el otro extremo del sistema escolar, en BUP, muchos alumnos $y$ alumnas dejan "Portobello» tras concluir la EGB. Se van a una "Comprehensive" del barrio para, en su opinión, poder conseguir "más» créditos sin tener que estudiar algunas asignaturas "inútiles» de BUP (Latín, Geografía y, desde luego, Religión, entre las que ellos siempre citan). La elección, en todo caso, se puede tomar como un indicador cierto de las preferencias y del sentido realista de tales jóvenes, de cara a su inmediato futuro en la sociedad inglesa.

Un segundo aspecto que resalta en el alumnado de «Portobello" es su anómala distribución por sexos: las chicas constituyen el $47,3 \%$ del alumnado. Lo anómalo aquí no es la cifra en sí, ya que ésta podría ser considerada normal en una distribución aleatoria de una población como la considerada, en torno a las 60.000 personas. Además, no hay forma de obtener estadísticas fiables del número y composición por sexos de la emigración española en Gran Bretaña, ni siquiera con un margen de un $5 \%$ de fiabilidad. Lo que llama la atención es que, por las tardes, en las clases Complementarias, hay más chicas que chicos. Lo que viene a mostrar que las chicas son enviadas a Colegios ingleses en mayor proporción que los chicos, probablemente con la intención de que su audibilidad, su "acento", no complique aún más la desventaja de partida que le supone su género. Por otro lado, el inglés «con buen acento» es un bien muy apreciado en una serie de profesiones "femeninas», tales como azafata, secretaria o intérprete. De cualquier forma, tales hechos apuntan a considerarlos como un primer aspecto de un conflicto que se da, a veces latente y otras abiertamente, en el seno de «Portobello".

\section{CONFLICTO CULTURAL/CONFLICTO LINGÜISTICO}

El conflicto apreciado en «Portobello» no es sino expresión del más amplio existente entre la cultura originaria, transmitida por los padres, y la adquirida del medio de residencia por los hijos, con la lengua como vehiculo privilegiado - pero no único-de expresión. Tal conflicto se da, así, en torno al sentido que las dos lenguas y los dos conjuntos de contenidos (currícula) tienen para el futuro del alumnado. Hasta ahora hemos visto algunas manifestaciones iniciales de tal conflicto, como el absentismo en las clases "complementarias», la «deserción» al final de la EGB o la "feminización" del Inglés, que, con ser ilustrativas, no son suficiente para una comprensión global del fenómeno. Es preciso pues, ensayar una explicación más global.

En general, hablar inglés sin acento es básico para una satisfactoria integración de estos chicos y chicas en «su» sociedad de nacimiento. Al mismo tiempo, deben conseguir las necesarias calificaciones y créditos para poder hacer operativa tal integración. Sin embargo, el origen familiar y la incertidumbre de sus padres entre la permanencia y el retorno, lastran ambos requerimientos, ya que les suponen compatibilizar el aprendizaje y la obtención de créditos (calificaciones) tanto en inglés como en español. En 
"Portobello", se observan una serie de expresiones de resistencia que pueden ser consideradas como producto de tal situación: murmullos y chistes en clase, uso del inglés como lengua de trabajo, una relativamente escasa utilización del comedor (de comida española)... Si bien tales manifestaciones pudieran ser tomadas como normales en otros centros escolares, especialmente las cuestiones de disciplina, la dirección de las mismas principalmente hacia aquello de origen español (profesores, asignaturas o comida) le confieren un carácter específico en "Portobello». Hay que tener en cuenta que una idea generalizada entre la colonia española en Londres es que allí se "aprende el inglés con acento» y se obtiene menos capital académico, «menos $\mathrm{O}$ levels», porque tienen que seguir asignaturas del currículum español de poca utilidad (latín o Religión) que les impide seguir un número mayor de créditos ( $\mathrm{O}$ levels) en el sistema inglés. Paralelamente, se da un proceso de identificación entre padres y profesorado de procedencia española, basado inicialmente en la coincidencia de lengua, origen y situación (estancia temporal en el país) y reforzado a posteriori por la similitud de las medidas disciplinarias (riñas y castigos), así como por la actitud con que responden a las muestras de resistencia. Por el contrario, las relaciones con el profesorado inglés parecen ser más distantes, marcadas por el formulismo del Miss o Mister en el trato. (Ver infra).

En todo caso, la búsqueda de una explicación del conflicto referido, debe hacerse analizando el día a día del alumnado de «Portobello» y, en general, de la vida del centro. Los diálogos en sus aulas, en los pasillos y patios o en la sala de profesores nos pueden ilustras convenientemente sobre lo que estamos llamado el conflicto de aprender entre dos culturas.

\section{Interacciones español-english}

En «Portobello», como colegio bilingüe que es, español e inglés comparten la formulación de la vida cotidiana en su interior ${ }^{2}$. Las dos lenguas se hablan en las aulas, en el patio y en el comedor, cada una con sus características y peculiaridades. Ambas lenguas son habladas por la totalidad del alumnado, con apenas excepciones y, a diferentes niveles de dominio y con bastantes excepciones, el profesorado: el núcleo de las interacciones lingüísticas en el colegio se lleva a cabo, pues, en inglés y en español.

Comenzando por el alumnado, su vida cotidiana está marcada por el dualismo. Incluso se podría pensar que la suya es una doble vida: español o gallego en casa, inglés en la calle, español en unas clases, inglés en otras: "Eastenders» en la BBC y «Los Santos Inocentes» en el vídeo. En todo caso, hacen uso de su dominio de ambas lenguas de acuerdo con las situaciones.

En casa, hablan con cada padre en su propia lengua, sea ésta el castellano, el inglés o el gallego. Con sus hermanos y hermanas, hablan inglés. Las situaciones más comunes pueden resumirse en las siguientes:

- "Yo hablo gallego con mis padres e inglés con mi hermana» (Chica de 2. ${ }^{\circ}$ de BUP).

- "Mi padre es andaluz y mi madre, gallega, y les hablo en español» (Chico, 8..$^{\circ}$ de EGB).

- "Hablo español a mi padre y hermano, e inglés a mi madre» (Chica de 2. de BUP). 
- «Mis padres son gallegos los dos, pero hablamos en inglés, porque como vemos la tele en inglés..." (Chico, $10^{\circ}$ de BUP).

- «Soy hija de padre español y madre inglesa, y hablo español con mi padre e inglés con mi madre. Con mi hermana hablo inglés" (Chica de $8 .^{\circ}$ de EGB).

- «Nosotros hablamos gallego, castellano e inglés, como salga» (Chico de 7.'de EGB).

El hecho de que hermanos y hermanas hablen inglés entre ellos, contribuye a la socialización de los más pequeños en este idioma. Por otra parte, ello denota la mayor cercanía e influencia de esta "segunda generación" de la cultura autóctona. Otro rasgo característico es la reserva del «otro» idioma, el menos o peor conocido por un tercero, como vía de comunicación confidencial o de escape. Esta alternativa es muy empleada en clạse.

En el colegio, consideran el inglés su lengua común de expresión, la que surge espontáneamente. Hablan inglés entre ellos y saben a quién y cuándo tienen que hablar español: al personal de origen español, sean profesores o personal de administración o servicios. Su dominio del inglés es mejor que el del español, tanto escrito como hablado. Tal dominio mejora con la edad, conforme tienen más contactos fuera del hogar familiar. Así, en «Portobello» se oye más español en la guarderia y en los primeros años de primaria que en secundaria: "se puede percibir en el patio, a la hora del recreo (...) hasta el final de la escuela primaria -7 años-, hablan español; cuando pasan al $4 .^{\circ}$ grado -equivalente a $3 .^{\circ}$ de EGB-, escogen el inglés» (Entrevista de campo con la Coordinadora de la Sección de Primaria Inglesa). Por tanto, dado que vienen de un ambiente español, lo que parece estar operando es un avance en el proceso de socialización en inglés, no sólo en la escuela, sino en la calles, en la sociedad. En este proceso, las referencias españolas se mantienen estabilizadas, dentro de una cultura nada viva. Esto significa, en la práctica, una pérdida en términos cualitativos y cuantitativos, mientras que el inglés, por otra parte, gana en sus vidas mayor espacio cada vez.

En clase, alumnos y alumnas hablan inglés como norma, pero con puntuales cambios a español. Una sistematización de sus interacciones podría ser la siguiente:

1. Trabajan, bromean y juegan en inglés. Como el profesor de Educación Física decía: «hablan inglés espontáneamente, tanto en el gimnasio como haciendo deporte en el patio. Si yo explico un juego, los de delante lo traducen en inglés a los de atrás que no oyen, o no lo entienden por mi acento andaluz". Asimismo, durante una clase de "Lengua», una chica corregía a otra al escribir "argentina» con mayúsculas, ya que («it's an adjective, not a noun") se trataba de un adjetivo, no del nombre de la nación. O en clase de "Ciencias»: "¿evaporación?... evaporation is like... the windows in the morning». $\mathrm{O}$, finalmente, también en otra clase de «Lengua», cuando otra chica preguntaba en alto si lo que había escrito la «señorita» en la pizarra era una «uay» (y griega).

2. Hacen continuas interrupciones en español, principalmente en situaciones cara a cara, en exclamaciones o cuando piden alguna cosa, como lo ilustran los ejemplos siguientes: 
— «I'll never take my mother there» — «Yo, tampoco» (English, 2. BUP, diálogo entre dos chicos).

- "Wait for me, ten paciencia» (Clase de «Sociales", 8. de EGB).

- «Qué la condensación es lo que forma las nubes? What do you say? - "That's true, lo acaba de decir la señorita» (Diálogó entre dos chicas en clase de "Ciencias» de $7 .^{\circ}$ de EGB).

- Frases como «Déjame un lápiz» o «Pobrecita», en conversaciones en inglés, se podrían añadir en gran número también.

3. Piden traducción o explicación de palabras a sus profesores, especialmente en el caso de español:

- "How do you say diseases in Spanish?" (Chica, 6. ${ }^{\circ}$ de EGB).

- "Alicia, ¿cómo se dice "operator» en español» (Chica, 7. EGB).

- «Señorita, ¿qué es un artesano?» (Chico de $60^{\circ}$ de EGB).

4. Se dirigen en inglés a quien está en el encerado, salvo cuando en la acción interviene directamente un profesor/a español. La situación contraria, en inglés, nunca se observó.

5. Se dirigen a cada profesor en su lengua, con el adecuado «Sir» o «Miss» o «Señorita» o «Profesor». En algunos casos, sin embargo, apean el tratamiento, llamándoles por sus nombres. En este sentido, esto se observó más frecuentemente con profesores españoles/as, mostrando una mayor «familiaridad».

6. En general, utilizan su bilingüismo como una ventaja ante el profesorado. Lo que fue calificado como «su ventaja» por un profesor inglés, comentado una de esas ocasiones, consiste en cambiar la conversación a la "otra» lengua cuando no quieren que el profesor se entere. Esto es posible porque no se requiere que los profesores sean bilingües, dada la dificultad de encontrarlos.

\section{Destreza y prácticas lingüisticas}

El análisis hecho hasta ahora de las interacciones bilingües en «Portobello» pretende fundamentar la afirmación de que español e «english», con primacía de este último, comparten la formulación de la vida del colegio. A partir de aquí es preciso plantearse qué inglés y qué español, de qué manera son utilizados ambos, cuáles son sus prácticas. Y ello no por una mera cuestión evaluativa de saber en qué aciertan o en qué fallan al hablar o escribir uno u otro idioma. Tampoco se trata de utilizar enfoques obsoletos, basados en síndromes carenciales o déficits sociales. Nada más alejado de la intención del estudio que una culpabilización de las víctimas: nadie debe culpar al alumnado de "Portobello" por su capital lingüístico. Se trata de ver si la doble destreza lingüística, fruto de una doble convivencia cultural, opera en la práctica como un elemento de discriminación y, por ello, de conflicto en relación a ambas comunidades. En definitiva, se trata de conocer el motivo por el cuál, mientras para algunos de sus moradores «se aprenden bien las dos lenguas en Portobello", para otros «existen muchas razones para apoyar la idea de que es más útil ir a una escuela inglesa. Una - continuaba diciendo un chico- es racismo. Si vas a la escuela bilingüe, desde luego que hablas inglés, pero pronunciado con acento español (...) Los que van a «Acton High School» pronuncian inglés correctamente » (Ci- 
tas sacadas de sendas redacciones presentadas por un grupo de $3 .^{\circ}$ de BUP-A Level).

Puede decirse que alumnos y alumnas de "Portobello" tienen unas características similares en el uso de ambas lenguas. Muestran un dominio de ambas, pero con interferencias comunes de elementos de una en otra. Además, y como antes ya se ha señalado, existe un predominio del inglés, principalmente coloquial e informal, sobre el español. Como explicación, sería ilustrador mencionar aquí la firme y contundente respuesta dada por una alumna de $3 .^{\circ}$ de BUP cuando se le preguntó la razón de ello: «He nacido y he vivido aquí toda mi vida». Un argumento de peso.

Seleccionando brevemente entre sus prácticas lingüísticas, se pueden ver los siguientes aspectos:

- Vocabulario: en casi todas las clases en español se daban interrupciones para preguntar el significado de algunas palabras: «cuenco", "progre», además de las ya citadas. Si bien en las clases en inglés raramente ocurrió, analizando redacciones o exámenes archivados, se encontraron numerosos ejemplos de traducciones literales, como «distracted» (de distraído), o «support» (de soportar).

- Ortografía: tendencia a escribir en inglés fonéticamente: «su ortografía - decía una profesora inglesa - refleja su pronunciación", dando ejemplos como «husban", "trank" o «sliper». En español, siguen las pautas corrientes en España: $\mathrm{v}$ y b, $\mathrm{g}$ y j, la h...

- Lenguaje formal: Otra común situación en las dos lenguas. Mientras en inglés les corrigen el uso del lenguaje informal en las redacciones, en español les dicen que no saben usar el Usted. En ambos casos, no obstante, se pueden estar reflejando las formas predominantemente informar de su cultura juvenil.

- Verbos: formas y tiempos verbales suponen algo problemático para la mayoría. Esto hace referencia no a traducciones literales como «dar el saco» por "despedir», sino al uso inadécuado de preposiciones o de estructuras como la doble negación, exportada del español al inglés o viceversa («Sabré hablar con ningún acento»).

En esencia y resumiendo, se trata de una interacción asimétrica: existe una continua interacción entre la dos lenguas, con mayor incidencia del inglés sobre el español. Sin embargo, cualquier apreciación de disfuncionalidad en su habla es inmediatamente entendida como algo negativo y objeto de preocupación. Ello afecta al fondo de su situación de manera directa: ser a no ser discriminados por su audibilidad. Jesús, un alumno de $2 .^{\circ}$ de BUP escribia: "when my parents came to England they had to learn English to find a job and to get around in this country. But they both speak a mixed up English, quite fluently. This is noticeable and Spanish people are harassed on the streets by some English people». Es pues el temor a que se pueda reproducir con ellos, la "segunda generación", lo que les pasó (y pasa) a sus padres, un elemento central en su conflicto bicultural. La audibilidad por el acento, el reconocimiento de la pertenencia a una minoría es temido por las chicas y chicos de "Portobello" como la causa de la discriminación y el racismo de la sociedad inglesa. 


\section{4}

\section{Profesores y teachers: una transmisión dual}

La transmisión bicultural en el Colegio bilingüe "Cañada-Blanch"se realiza por un cuerpo de profesores dual en cuanto a su origen. Profesoras y profesores de procedencia inglesa o española han tenido una formación distinta, han sido seleccionados de manera distinta (a través de un anuncio en la prensa los ingleses y mediante un proceso meritocrático dentro del cuerpo funcionarial, los españoles) y reciben sueldos muy desiguales (los españoles, el doble). Pero además se da una distinción espacial añadida entre los dos colectivos: a pesar de existir una sala de profesores común, ésta es utilizada habitualmente sólo por los españoles, mientras que los ingleses se reúnen en los departamentos. La sala de profesores es la «zona española», a ella no van los ingleses, salvo para acciones concretas y sin apenas traspasar la puerta, «porque se fuma» (negro, por supueso). Pero si el claustro hispano levanta la "cortina de humo" para esparcirse y liberar tensiones, por su parte, profesores y profesoras ingleses anteponen la barrera de la «ética protestante» del trabajo para definir su «english area». Allí no se fuma - sólo de vez en cuando-, se trabaja para evitar llevarse tarea a casa.

Tal separación espacial no es una mera descripción antropológica, sino que puede ser tomada como epítome de otra de más trascendencia. De lo que se trata es de plantear si tal cortina o barrera no se levanta también entre los contenidos que se transmiten y entre las prácticas docentes. Del trabajo de campo se pueden ver sendos grupos como «tipos ideales» o modelos pedagógicos. Así, mientras en el grupo español predomina la explicación oral y global a toda la clase, la llamada lección magistral, en el inglés se tiende a la enseñanza personalizada ("pupil centred») característica de la educación para la clase obrera inglesa. Los dos currícula, si operan conjuntamente en la programación general del colegio, en la práctica lo hacen separadamente, a través de las propias prácticas del profesorado. Alumnas y alumnos reciben y reconocen así diferentes contenidos, en diferentes idiomas y mediante diferentes métodos. Los diferentes programas expresan de forma simbólica, pero precisa, la separación cultural. Quizá un ejemplo anecdótico, pero ilustrativo, pueda ser el tema de la «Spanish Armada», central en «Social Studies» y apenas considerado en Historia. De más enjundia sería un análisis comparativo entre asignaturas paralelas, como el caso de las "Matemáticas", donde la asignatura española, pese a gozar de alta estima es mal llevada por los escolares y la inglesa, pese a estar devaluada, es bien «soportada» en las aulas. Igualmente, al observar las bilbliotecas de cada aula, se puede ver como mientras en la de "Lengua» dominaban los diccionarios y libros de texto, en la «English classroom», casi todos los volúmenes eran de literatura. Cálculo vs. fichas o reglas gramaticales vs. escritura libre, podrían ser expresiones paradigmáticas de tal dualidad.

Usando a Bernstein (1975) de nuevo, puede decirse que existe una «clasificación» fuerte $(+C)$ entre los dos sistemas educativos operando en "Portobello», a pesar incluso de las instancias de coordinación (Jefatura de Estudios y Coordinaciones de Sección). Tal separación contribuye a la dicotomización de las dos culturas, sin facilitar su integración. Es más, tal dicotomía reproduce el conflicto entre ellas. La cultura parentalmente dotada es más estricta y más distante en espacio y tiempo. La autóctona, producto del entorno, es más permisiva y operativa, más cercana a la vida de los chi- 
cos y chicas estudiando en "Portobello". Se trata de un conflicto entre el pasado y el futuro localizado en un presente dual. Es la tensión entre el legado parental y su porvenir en medio de la incertidumbre: dado que no están seguros de donde van a vivir, tienen que cargar con los dos currículos con igual énfasis para poder conseguir el capital necesario para vivir en ambas situaciones. Una ambigüedad potenciada por los padres, para los que asegurar el doble futuro de sus hijos/as es fundamental para mantener abierto el suyo propio.

Esa carga dificulta, a más de hacer penoso, el proceso de aprendizaje y de consecución de más y mejores calificaciones. Las respuestas en forma de resistencia que dan al alto coste del dualismo se pueden presentar como reacciones ante el conflicto creado por la incertidumbre de sus padres. De ahí su orientación hacia los elementos de esa procedencia, que se veía más arriba.

En todo caso, dentro de las manifestaciones de resistencia no fue posible establecer con precisión aquéllas de carácter transformador de las de carácter reproductivo. Aunque tampoco estaba claro que existiesen, quizá una línea de investigación hubiese ido por el estudio de un grupo especial de 10 chicos y 3 chicas, segregado del resto y etiquetados como los «leavers». Curiosamente, era el único grupo o clase con nombre inglés en el centro, y no simplemente porque la posibilidad de segregación y repetición de curso no se contempla en el sistema español. Mayores de 16 años, arrastrando problemas de disciplina de cursos precedentes y sin haber terminado la EGB, habían sudo puestos juntos con un currículum específico que les pudiese aportar el máximo de créditos ( $\mathrm{O}$ levels) con que «dejar» (leave) el colegio hacia la sociedad inglesa. Dicho grupo podría haber sido seguramente el más indicado para profundizar en el conocimiento del proceso de aprender entre dos culturas $\longrightarrow$ entre dos «subtipos de códigos educativos» (Bernstein, 1975) y ver qué elementos reproductivos y transformadores se pueden dar en el mismo. La presunción descansa en que estos chicos y chicas eran, por una parte, representantes cualificados del fracaso de tal aprendizaje $y$, por otra, los que se habían enfrentado con el desajuste bicultural de forma más patente. Por ello su etiquetado (Hargreaves, 1976) y separación del resto. Sin embargo, tal grupo formaba parte de aquello que Bárbara Stein llama «lo sagrado» de toda investigación (en Hammersley y Atkinson, 1983), aquello que se oculta o, al menos, se camufla y no se enseña del todo. Por ello, apenas se pudo observar a los «leavers» y, en consecuencia, no se pueden aportar demasiadas claves sobre lo planteado. Quede pues el análisis de estas formas de resistencia sólo como una posibilidad de explicación, a desarrollar por futuros estudios de ese conflicto que es aprender entre dos culturas, de los problemas de adaptarse a, o ser adaptados por, una sociedad en la que se pertenece a una cultura minoritaria y discriminada.

\section{Notas}

1 Una formulación más detallada de estas consideraciones metodológicas puede hallarse en la memoria de mi «Dissertation*, Learning between two cultures. A case-study of bilingual schooling for inmigrants' children, especialmente el capítulo 1 y sus referencias biblio- 
gráficas. No obstante, una primera aproximación se puede hallar en HAMMERSLEY y WOODS (1976), HAMMERSLEY y ATKINSONS (1983) y BURGESS (1982 y 1984).

2 Aunque oficialmente bilingüe, «Portobello» es, en realidad, trilingüe: el gallego, la lengua hablada en casa por un gran número de familias, puede oírse frecuentemente en clase, en los pasillos o en el patio, pero no así en la sala de profesores. Sin embargo, su uso está reducido a interacciones entre alumnos/as a nivel privado. Cuando aparece en clase públicamente, provoca situaciones cómicas o es ridiculizado. Se podría decir que "Portobello" contribuye en este sentido a la dominación del castellano sobre otras lenguas periféricas.

\title{
Referencias
}

BALL, S. (1984). «Beachside Reconsidered: Reflections on a Methodological Aprenticeship", en Burgess (1984), 69-96.

BERNSTEIN, B. (1975). «Class, Codes and Control». Vol. III. "Towards a Theory of Educational Transmissions», Routledge and Kegan Paul, Londres.

Bourdieu, P. y PASSERon, J. C. (1977). «La Reproducción. Elementos para una teoría del sistema de enseñanza", Laia, Barcelona.

BURGESS, R. G. (ed.) (1982). «Field Research: a sourcebook and field manual». Allen y Unwin, Londres.

BuRgess, R. G. (ed.) (1984). «The Research Process in Educational Settings», Falmer Press, Londres.

Denzin, N. J. (1970). «The Research Act", Butterworth, Londres.

Hammersley, M. y AtKInSON, P. (1983). «Ethnography. Principles in Practice», Tavistock, Londres.

Hammersley, M. y Woods, P. (eds.) (1976). "The Process of Schooling», Routledge y Kegan Paul, Londres.

HARGREAVES, D. H. (1976). "Reactions to Labelling", en Hammersley y Woods (eds.), 201-208.

\section{Aprender entre dos culturas: Estudio de un caso de escolarización bilingüe para hijos de emigrantes.}

\author{
A. Guerrero. \\ CLEE, 1990, 7-8, pp. 145-156.
}

Resumen: A lo largo de este artículo se presentan una serie de elementos que aparecieron durante un estudio etnográfico realizado en el Colegio bilingüe para bijos de emigrantes españoles existente en Londres, más conocido como «Portobello». El texto es un ensayo de interpretación de los datos aparecidos en el trabajo de campo y que componen el contexto en que se desarrolla la transmisión bi-cultural, el aprendizaje entre dos culturas asimétricas y el significado de ambas tanto para chicos y chicas, como para sus padres. Entre otros contenidos, se analiza el valor del SEE como agente de cambio del capital académico; la contribución conflictiva que la audibilidad (acento) y la incertidumbre tienen en tal aprendizaje; $y$ de la dualidad y dicotomía de lenguas y curricula, enraizados en un doble cuerpo de profesorado y un doble contenido cultural. Al final, se apunta hacia el análisis de las respuestas en forma de resistencia como una interesante y posible vía de explicación complementaria de ese conflicto y sus salidas.

Datos sobre el autor: Antonio Guerrero es MSC en Sociología por el Instituto de Educación de la Universidad de Londres, donde ha trabajado en temas de multiculturalismo y bilingüismo. En la actualidad es profesor de Sociología en la Escuela Universitaria de Formación de Profesorado, donde investiga en temas de profesionalismo y sindicación del profesorado.

Dirección: Universidad Complutense de Madrid, Escuela Universitaria de Formación de Profesorado María Jiménez. Avda. de Islas Filipinas, 3, 28071, Madrid.

(C) De todos los artículos. Deberá solicitarse por escrito autorización de CL\&E y de los autores para el uso en forma de facsímil, fotocopia o cualquier otro medio de reproducción impresa. CL\&E se reserva el derecho de interponer las acciones legales necesarias en aquellos casos en que se contravenga la ley de derechos de autor. 